\title{
Existence and stability results for Hilfer-Katugampola-type fractional implicit differential equations with nonlocal con- ditions
}

\author{
Ahmad Y. A. Salamooni*, D. D. Pawar \\ School of Mathematical Sciences, Swami Ramanand Teerth Marathwada University, Nanded-431606, India.
}

\begin{abstract}
This article contains a new discussion for Hilfer-Katugampola-type fractional derivative. We establish an existence and uniqueness results of Hilfer-Katugampola-type fractional derivative for implicit differential equations with the help of Schaefer's fixed point theorem and Banach contraction principle. Also, we use the Gronwall's lemma for singular kernels to prove the Ulam-Hyers-Rassias stability results. Further, the examples are given to illustrate our main results.
\end{abstract}

Keywords: Hilfer-Katugampola-type fractional derivative, implicit differential equation, Schaefer's fixed point theorem, existence, uniqueness, Ulam stability.

2020 MSC: 34A08, 26A33, 34B10, 34A12.

(C)2021 All rights reserved.

\section{Introduction}

Fractional differential equations come to light in many scientific disciplines, engineering as the mathematical modeling of systems processes in the fields of chemistry, physics, electrodynamics of complex medium, aerodynamics, polymer rheology. In the last few years there has been considerable development on the ordinary and partial fractional differintegrals equations, see $[2,3,5,11-13,15,25,32,33]$ and references therein. There are many general definitions which interpolates to other differintegrals for particular values of parameters reported in the literature, but the most familiar definitions are the famous definitions of Riemann-Liouville, Caputo, Hadamard and Hilfer [15, 24, 28]. Recently, Katugampola has introduced a generalized form of the Riemann-Liouville fractional differential derivatives and integrals [22, 23]. Thereafter, OLiveira et al. [27], have established a more generalized form of Katugampola fractional derivative which is Hilfer-Katugampola. This formulation is an interpolation for the popular definitions of the Hilfer, Hilfer-Hadamard, Caputo-Hadamard and Riemann Liouville fractional derivatives, etc, we refer the reader to [27].

In 1940, Ulam gave a wide ranging talk before the mathematics club of the Wisconsin university in which he discussed several important unsolved problems $[35,36]$ and he raised the question concerning

\footnotetext{
*Corresponding author

Email addresses: ayousss83@gmail.com (Ahmad Y. A. Salamooni), dypawar@yahoo.com (D. D. Pawar)

doi: $10.22436 /$ jnsa.014.03.02
}

Received: 2020-06-24 Revised: 2020-08-07 Accepted: 2020-08-17 
the homomorphism stability. In the next years, Hyers positively answered the question of Ulam's partially [16]. Moreover, a generalized was obtained by Rassias for the Hyers results [29, 30]. And since 1940, the mathematics authors have been interested to studied the Ulam-type stability problems [4, 26, 31]. In last few decades, Jung extended the previously mentioned stability results, see [19-21]. The Ulam stability analysis is very useful in lot of applications, such as optimization, numerical analysis, etc, for more detailed study of Ulam-type stability with different approaches see [17, 38, 39]. Some recent work on stability of differential equations (or their solutions) with variety of initial or boundary conditions are reported in $[1,6-10,18,37]$.

Vivek et al. [37] in 2018, have obtained some existence and stability results for Hilfer fractional implicit differential equations with nonlocal conditions of the form

$$
\begin{cases}D_{0^{+}}^{\alpha, \beta} \chi(t)=\varphi\left(t, x(t), D_{0^{+}}^{\alpha, \beta} \chi(t)\right), & t \in[0, T], 0<\alpha<1,0 \leqslant \beta \leqslant 1, \\ I_{0^{+}}^{1-\gamma} x(0)=\sum_{i=1}^{n} \eta_{i} x\left(\xi_{i}\right), & \alpha \leqslant \gamma=\alpha+\beta(1-\alpha)<1, \quad \xi_{i} \in(0, T),\end{cases}
$$

where $D_{0^{+}}^{\alpha, \beta}$ is the Hilfer fractional derivative of order $\alpha$ and type $\beta$.

Bhairat [9], studied the existence and stability of fractional differential equations involving generalized Katugampola derivative

$$
\begin{cases}{ }_{\rho} \mathrm{D}_{0^{+}}^{\alpha, \beta} \chi(t)=\varphi(t, x(t)), & t \in \Omega, 0<\alpha<1,0 \leqslant \beta \leqslant 1 \\ { }_{\rho} \mathrm{I}_{0^{+}}^{1-\gamma} \chi(0)=x_{0}, & \gamma=\alpha+\beta(1-\alpha), \quad x_{0} \in \mathbb{R}\end{cases}
$$

where $\rho>0$ and ${ }_{\rho} D_{0^{+}}^{\alpha, \beta}$ is the Hilfer-Katugampola-type fractional derivative of order $\alpha$ and type $\beta$.

Ibrahim et al. [18], have discuss the existence, uniqueness and stability results for a general class of Langevin equations with two Hilfer-Katugampola fractional derivatives as follows of the form

$$
\begin{cases}{ }_{\rho} \mathrm{D}^{\alpha_{1}, \beta}\left({ }_{\rho} \mathrm{D}^{\alpha_{2}, \beta}+\mathrm{c}\right) \chi(\mathrm{t})=\varphi(t, x(t)), & t \in(a, b] \\ \rho{ }^{1-\gamma} \chi(a)=x_{a}, & \gamma=\left(\alpha_{1}+\alpha_{2}\right)(1-\beta)+\beta\end{cases}
$$

where $\rho>0$ and ${ }_{\rho} D^{\alpha_{1}, \beta},{ }_{\rho} D^{\alpha_{2}, \beta}$ are Hilfer-Katugampola fractional differential operator of orders $\alpha_{1}, \alpha_{2}$, respectively and type $\beta$.

The aim of this article is to study the implicit differential equation with nonlocal condition involving Hilfer-Katugampola-type fractional derivative of the following form

$$
\begin{cases}{ }_{\rho} \mathrm{D}_{0^{+}}^{\alpha, \beta} \chi(t)=\varphi\left(t, x(t),{ }_{\rho} \mathrm{D}_{0^{+}}^{\alpha, \beta} \chi(t)\right), & t \in \mathbb{I}:=[0, \mathrm{~T}], \quad 0<\alpha<1,0 \leqslant \beta \leqslant 1, \\ { }_{\rho} \mathrm{I}_{0^{+}}^{1-\gamma} \chi(0)=\sum_{i=1}^{n} \xi_{i} x\left(\eta_{i}\right), & \alpha \leqslant \gamma=\alpha+\beta(1-\alpha)<1,\end{cases}
$$

where $\rho>0$ and ${ }_{\rho} D_{0^{+}}^{\alpha, \beta}$ is the Hilfer-Katugampola-type fractional derivative of order $\alpha$ and type $\beta,{ }_{\rho} \mathrm{I}_{0^{+}}^{1-\gamma}$ is the Katugampola fractional integral of order $1-\gamma, \eta_{i} \in(0, T), \xi_{i} \in \mathbb{R}$, for all $i=1,2, \ldots, n, \eta_{1} \leqslant \eta_{2} \leqslant$ $\cdots \leqslant \eta_{n}$ and here we let $\mathbb{R}$ be a Banach space, $\varphi: \mathbb{I} \times \mathbb{R} \times \mathbb{R} \rightarrow \mathbb{R}$ is a continuous function.

The rest of this paper is structured as follows: Section 2 contains some fundamental concepts on Hilfer-Katugampola-type fractional derivative. In Section 3, we present our main result of existence and uniqueness by using Schaefer's fixed point theorem and Banach contraction principle. Further, we discuss stability analysis of Hilfer-Katugampola-type fractional derivative. The last section contains some examples to illustrate our main results.

\section{Preliminaries}

In this section, we introduce some basic notations, definitions and lemmas regarding the theory of fractional differential equations, which will be used later in this paper, to obtain our main results.

In the light of the [27, Definition 3] we have the following definition. 
Definition 2.1. Let $C[\mathbb{I}, \mathbb{R}]$ is a Banach space of continuous functions $\varphi$ from $\mathbb{I}$ into $\mathbb{R}$ with the norm $\|\varphi\|_{C}=\max \{|\varphi(t)|: t \in \mathbb{I}\}$ and the Lebesgue space $L^{1}(\mathbb{I})$ of integrable functions $\varphi: \mathbb{I} \rightarrow \mathbb{R}$ with the norm $\|\varphi\|_{1}=\int_{0}^{T}|\varphi(\tau)| d \tau$. For $\rho>0$ and $0 \leqslant \gamma<1$, the weighted space $C_{\gamma, \rho}[\mathbb{I}, \mathbb{R}]$ of continuous functions $\varphi$ on $(0, T]$ is defined by

$$
\mathrm{C}_{\gamma, \rho}[\mathbb{I}, \mathbb{R}]=\left\{\varphi:(0, \mathrm{~T}] \rightarrow \mathbb{R}:\left[\left(\mathrm{t}^{\rho} / \rho\right)\right]^{\gamma} \varphi(\mathrm{t}) \in \mathrm{C}[\mathbb{I}, \mathbb{R}]\right\}
$$

with the norm

$$
\|\varphi\|_{C_{\gamma, \rho}}=\left\|\left[\left(t^{\rho} / \rho\right)\right]^{\gamma} \varphi(t)\right\|_{C}=\max _{t \in \mathbb{I}}\left|\left[\left(t^{\rho} / \rho\right)\right]^{\gamma} \varphi(t)\right|,
$$

where $\mathrm{C}_{0, \rho}[\mathbb{I}, \mathbb{R}]=\mathrm{C}[\mathbb{I}, \mathbb{R}]$. Obviously,

$$
\mathrm{C}_{\gamma, \delta_{\rho}}^{n}[\mathbb{I}, \mathbb{R}]=\left\{\varphi \in \mathrm{C}_{\delta_{\rho}}^{n-1}[\mathbb{I}, \mathbb{R}]: \delta_{\rho}^{n} \varphi \in \mathrm{C}_{\gamma, \rho}[\mathbb{I}, \mathbb{R}]\right\}
$$

is the Banach space with the norm

$$
\|\varphi\|_{C_{\gamma, \delta \rho}^{n}}=\sum_{k=0}^{n-1}\left\|\delta_{\rho}^{k} \varphi\right\|_{C}+\left\|\delta_{\rho}^{n} \varphi\right\|_{C_{\gamma, \rho}}, \quad\|\varphi\|_{C_{\gamma, \delta \rho}^{n}}=\sum_{k=0}^{n} \max _{t \in \mathbb{I}}\left|\delta_{\rho}^{k} \varphi(t)\right|, \quad n \in \mathbb{N} .
$$

For $\mathrm{n}=0$, we have

$$
\mathrm{C}_{\gamma, \delta_{\rho}}^{0}=\mathrm{C}_{\gamma, \rho}[\mathbb{I}, \mathbb{R}] .
$$

Also, for $0<\alpha<1,0 \leqslant \beta \leqslant 1$, we define the following spaces

$$
\mathrm{C}_{1-\gamma, \rho}^{\alpha, \beta}[\mathbb{I}, \mathbb{R}]=\left\{\varphi \in \mathrm{C}_{1-\gamma, \rho}[\mathbb{I}, \mathbb{R}],{ }_{\rho} \mathrm{D}_{0^{+}}^{\alpha, \beta} \varphi \in \mathrm{C}_{1-\gamma, \rho}[\mathbb{I}, \mathbb{R}]\right\},
$$

and

$$
\mathrm{C}_{1-\gamma, \rho}^{\gamma}[\mathbb{I}, \mathbb{R}]=\left\{\varphi \in \mathrm{C}_{1-\gamma, \rho}[\mathbb{I}, \mathbb{R}],{ }_{\rho} \mathrm{D}_{0^{+}}^{\gamma} \varphi \in \mathrm{C}_{1-\gamma, \rho}[\mathbb{I}, \mathbb{R}]\right\} .
$$

Clearly,

$$
\mathrm{C}_{1-\gamma, \rho}^{\gamma}[\mathbb{I}, \mathbb{R}] \subset \mathrm{C}_{1-\gamma, \rho}^{\alpha, \beta}[\mathbb{I}, \mathbb{R}] .
$$

Definition 2.2 ([27]). Let $\Omega=(0, \mathrm{~T}]$ and $\varphi:(0, \infty) \rightarrow \mathbb{R}$, the Katugampola fractional integral $\rho_{0^{+}}^{\alpha} \varphi$ of order $\gamma \in \mathbb{C}(\mathfrak{R}(\alpha)>0)$ is defined for $\rho>0$ as

$$
\left({ }_{\rho} \mathrm{I}_{0^{+}}^{\alpha} \varphi\right)(\mathrm{t})=\frac{\rho^{1-\alpha}}{\Gamma(\alpha)} \int_{0}^{t} \frac{\tau^{\rho-1} \varphi(\tau)}{\left(t^{\rho}-\tau^{\rho}\right)^{1-\alpha}} d \tau, \quad(t>0),
$$

and the corresponding Katugampola fractional derivative ${ }_{\rho} \mathrm{D}_{0^{+}}^{\alpha} \varphi$ is defined as

$$
\left({ }_{\rho} D_{0^{+}}^{\alpha} \varphi\right)(t):=\left(t^{1-\rho} \frac{d}{d t}\right)^{n}\left({ }_{\rho} I_{0^{+}}^{n-\alpha} \varphi\right)(t)=\frac{\rho^{\alpha-n+1}}{\Gamma(n-\alpha)}\left(t^{1-\rho} \frac{d}{d t}\right)^{n} \int_{0}^{t} \frac{\tau^{\rho-1} \varphi(\tau)}{\left(t^{\rho}-\tau^{\rho}\right)^{\alpha-n+1}} d \tau, \quad(t>0),
$$

Definition 2.3 ([27]). Let $0<\alpha<1, \quad 0 \leqslant \beta \leqslant 1, \quad \varphi \in \mathrm{C}_{1-\gamma, \rho}[0, \mathrm{~T}]$. The Hilfer-Katugampola fractional derivative ${ }_{\rho} D^{\alpha, \beta}$ of order $\alpha$ and type $\beta$ of $\varphi$ is defined as

$$
\begin{aligned}
\left({ }_{\rho} D^{\alpha, \beta} \varphi\right)(t) & =\left({ }_{\rho} I^{\beta(1-\alpha)}\left(t^{1-\rho} \frac{d}{d t}\right){ }_{\rho} I^{(1-\alpha)(1-\beta)} \varphi\right)(t) \\
& =\left({ }_{\rho} I^{\beta(1-\alpha)}\left(\delta_{\rho}\right){ }_{\rho} I^{(1-\alpha)(1-\beta)} \varphi\right)(t) ; \quad \gamma=\alpha+\beta(1-\alpha),
\end{aligned}
$$

where ${ }_{\rho} \mathrm{I}^{(.)}$is the Katugampola fractional integral given in Definition 2.2. 
Lemma 2.4 (Schaefer fixed point Theorem, [14]). Let $\mathrm{E}$ be a Banach space and $\mathrm{K}: \mathrm{E} \rightarrow \mathrm{E}$ is a continuous and compact mapping. If,

$$
X=\{x \in E: x=\omega K(x) \text { for some } \omega \in[0,1]\}
$$

is a bounded set, then $\mathrm{K}$ has a fixed point.

Lemma 2.5 ([27]). Let $0<\alpha<1,0 \leqslant \beta \leqslant 1, \gamma=\alpha+\beta(1-\alpha)$, and assume that $\varphi(t, x(t)) \in C_{1-\gamma, \rho}[0, T]$ where $\varphi:(0, T] \times \mathbb{R} \rightarrow \mathbb{R}$ be a function for any $x \in C_{1-\gamma, \rho}[0, T]$. If $x \in C_{1-\gamma, \rho}^{\gamma}[0, T]$, then $x$ satisfies (1.1), if and only if $x$ satisfies the second kind Volterra fractional integral equation

$$
x(t)=\frac{x_{0}}{\Gamma(\gamma)}\left(t^{\rho} / \rho\right)^{\gamma-1}+\frac{\rho^{1-\alpha}}{\Gamma(\alpha)} \int_{0}^{t} \frac{\tau^{\rho-1} \varphi(\tau, x(\tau))}{\left(t^{\rho}-\tau^{\rho}\right)^{1-\alpha}} d \tau, \quad(t>0) .
$$

Lemma 2.6 ([27]). Let $\mathrm{t}>0,{ }_{\rho} \mathrm{I}_{0+}^{\alpha}$ and ${ }_{\rho} \mathrm{D}_{0^{+}}^{\alpha}$, as defined in Definition 2.2. Then for $\alpha \geqslant 0, \beta>0$, we have

$$
\left(\rho \mathrm{I}_{0^{+}}^{\alpha}\left(\tau^{\rho} / \rho\right)^{\beta-1}\right)(\mathrm{t})=\frac{\Gamma(\beta)}{\Gamma(\alpha+\beta)}\left(\mathrm{t}^{\rho} / \rho\right)^{\alpha+\beta-1},
$$

and for $0<\alpha<1$

$$
\left({ }_{\rho} D_{0^{+}}^{\alpha}\left(\tau^{\rho} / \rho\right)^{\alpha-1}\right)(t)=0 .
$$

Lemma 2.7 ([22]). Let $0<a<b<\infty, 1 \leqslant p \leqslant \infty, \alpha, \beta>0$ and let $(c \leqslant \rho) \rho, c \in \mathbb{R}$. Then, for $\varphi \in X_{c}^{p}(a, b)$ the following semigroup property holds

$$
\left({ }_{\rho} \mathrm{I}_{0^{+}}^{\alpha} \mathrm{I}_{0^{+}}^{\beta} \varphi\right)(\mathrm{t})=\left({ }_{\rho} \mathrm{I}_{0^{+}}^{\alpha+\beta} \varphi\right)(\mathrm{t}),
$$

and (see [14])

$$
\left({ }_{\rho} \mathrm{D}_{0^{+} \rho}^{\beta} \mathrm{I}_{0^{+}}^{\beta} \varphi\right)(\mathrm{t})=\varphi(\mathrm{t}) .
$$

In the light of the lemmas (Lemma 7, Lemma 9 , and Lemma 12, see [27]), we have the following lemmas.

Lemma 2.8. Assume that $0<\alpha<1,0 \leqslant \gamma<1, \rho>0$. If $\varphi \in \mathrm{C}_{\gamma, \rho}[\mathbb{I}, \mathbb{R}]$ and ${ }_{\rho} \mathrm{I}_{0+}^{1-\beta} \varphi \in \mathrm{C}_{\gamma, \rho}^{1}[\mathbb{I}, \mathbb{R}]$, then

$$
\left({ }_{\rho} \mathrm{I}_{0^{+}}^{\alpha}{ }_{\rho} \mathrm{D}_{0^{+}}^{\alpha} \varphi\right)(\mathrm{t})=\varphi(\mathrm{t})-\frac{\left({ }_{\rho} \mathrm{I}_{0^{+}}^{1-\alpha} \varphi\right)(0)}{\Gamma(\alpha)}\left(\mathrm{t}^{\rho} / \rho\right)^{\alpha-1}, \quad \text { for all } \mathrm{t} \in \mathbb{I} .
$$

Lemma 2.9. Assume that $\alpha>0,0 \leqslant \gamma<1, \rho>0$ and $\varphi \in \mathrm{C}_{\gamma, \rho}[\mathbb{I}, \mathbb{R}]$. If $\gamma<\alpha$, then

$$
\left({ }_{\rho} \mathrm{I}_{0^{+}}^{\alpha} \varphi\right)(0)=\lim _{\mathrm{t} \rightarrow 0^{+}}\left({ }_{\rho} \mathrm{I}_{0^{+}}^{\alpha} \varphi\right)(\mathrm{t})=0 .
$$

Lemma 2.10. Assume that $0<\alpha<1,0 \leqslant \beta \leqslant 1, \rho>0$ and $\gamma=\alpha+\beta(1-\alpha)$. If $\varphi \in C_{1-\gamma, \rho}^{\gamma}[\mathbb{I}, \mathbb{R}]$, then

$$
{ }_{\rho} \mathrm{I}_{0^{+}}^{\gamma}{ }_{\rho} \mathrm{D}_{0^{+}}^{\gamma} \varphi={ }_{\rho} \mathrm{I}_{0^{+}}^{\alpha}{ }_{\rho} \mathrm{D}_{0^{+}}^{\alpha, \beta} \varphi, \quad{ }_{\rho} \mathrm{D}_{0^{+}}^{\gamma}{ }_{\rho} \mathrm{I}_{0^{+}}^{\alpha} \varphi={ }_{\rho} \mathrm{D}_{0^{+}}^{\beta(1-\alpha)} \varphi .
$$

Now, in the following lemma we establish an important equivalent mixed-type Volterra fractional integral equation for our problem (1.2), which we will utilize later in our main results.

Lemma 2.11. Let $0<\alpha<1,0 \leqslant \beta \leqslant 1, \gamma=\alpha+\beta(1-\alpha)$, and assume that $\varphi\left(t, x(t),{ }_{\rho} D_{0^{+}}^{\alpha, \beta} x(t)\right) \in$ $\mathrm{C}_{1-\gamma, \rho}[\mathbb{I}, \mathbb{R}]$ where $\varphi: \mathbb{I} \times \mathbb{R} \times \mathbb{R} \rightarrow \mathbb{R}$ be a function for any $x \in \mathrm{C}_{1-\gamma, \rho}[\mathbb{I}, \mathbb{R}]$. If $x \in \mathrm{C}_{1-\gamma, \rho}^{\gamma}[\mathbb{I}, \mathbb{R}]$, then $x$ satisfies (1.2), if and only if $x$ satisfies the mixed-type Volterra fractional integral equation

$$
x(t)=\frac{\Psi \rho^{1-\alpha}}{\Gamma(\alpha)}\left(t^{\rho} / \rho\right)^{\gamma-1} \sum_{i=1}^{n} \xi_{i} \int_{0}^{\eta_{i}} \frac{\tau^{\rho-1} \Lambda_{x}(\tau)}{\left(\eta_{i}^{\rho}-\tau^{\rho}\right)^{1-\alpha}} d \tau+\frac{\rho^{1-\alpha}}{\Gamma(\alpha)} \int_{0}^{t} \frac{\tau^{\rho-1} \Lambda_{x}(\tau)}{\left(t^{\rho}-\tau^{\rho}\right)^{1-\alpha}} d \tau,
$$


where

$$
\Psi:=\frac{1}{\Gamma(\gamma)-\sum_{i=1}^{n} \xi_{i}\left(\eta_{i}^{\rho} / \rho\right)^{\gamma-1}}, \quad \text { if } \quad \Gamma(\gamma) \neq \sum_{i=1}^{n} \xi_{i}\left(\eta_{i}^{\rho} / \rho\right)^{\gamma-1}
$$

and

$$
\Lambda_{\chi}(t):={ }_{\rho} D_{0^{+}}^{\alpha, \beta} \chi(t)=\varphi\left(t, x(t), \Lambda_{\chi}(t)\right)
$$

Proof. Firstly, we will prove the necessary condition. According to Lemma 2.5, we can express the solution of the problem (1.2) as follows

$$
x(t)=\frac{\rho_{0^{+}}^{1-\gamma} \chi(0)}{\Gamma(\gamma)}\left(t^{\rho} / \rho\right)^{\gamma-1}+\frac{\rho^{1-\alpha}}{\Gamma(\alpha)} \int_{0}^{t} \frac{\tau^{\rho-1} \Lambda_{\chi}(\tau)}{\left(t^{\rho}-\tau^{\rho}\right)^{1-\alpha}} d \tau .
$$

Now, by using the substitution $t=\eta_{i}$ in (2.3), we have

$$
x\left(\eta_{i}\right)=\frac{\rho^{1-\gamma} I^{+} \chi(0)}{\Gamma(\gamma)}\left(\eta_{i}^{\rho} / \rho\right)^{\gamma-1}+\frac{\rho^{1-\alpha}}{\Gamma(\alpha)} \int_{0}^{\eta_{i}} \frac{\tau^{\rho-1} \Lambda_{\chi}(\tau)}{\left(\eta_{i}^{\rho}-\tau^{\rho}\right)^{1-\alpha}} d \tau
$$

by multiply $\xi_{i}$ on both sides of the equation (2.4), we get

$$
\xi_{i} x\left(\eta_{i}\right)=\frac{\rho I_{0^{+}}^{1-\gamma} \chi(0)}{\Gamma(\gamma)} \xi_{i}\left(\eta_{i}^{\rho} / \rho\right)^{\gamma-1}+\frac{\rho^{1-\alpha}}{\Gamma(\alpha)} \xi_{i} \int_{0}^{\eta_{i}} \frac{\tau^{\rho-1} \Lambda_{x}(\tau)}{\left(\eta_{i}^{\rho}-\tau^{\rho}\right)^{1-\alpha}} d \tau
$$

Next, by using the initial value given in problem (1.2), we have

$$
{ }_{\rho} I_{0^{+}}^{1-\gamma} \chi(0)=\sum_{i=1}^{n} \xi_{i} x\left(\eta_{i}\right)=\frac{\rho I_{0^{+}}^{1-\gamma} x(0)}{\Gamma(\gamma)} \sum_{i=1}^{n} \xi_{i}\left(\eta_{i}^{\rho} / \rho\right)^{\gamma-1}+\frac{\rho^{1-\alpha}}{\Gamma(\alpha)} \sum_{i=1}^{n} \xi_{i} \int_{0}^{\eta_{i}} \frac{\tau^{\rho-1} \Lambda_{x}(\tau)}{\left(\eta_{i}^{\rho}-\tau^{\rho}\right)^{1-\alpha}} d \tau,
$$

which implies that

$$
{ }_{\rho} I_{0^{+}}^{1-\gamma} \chi(0)\left(\Gamma(\gamma)-\sum_{i=1}^{n} \xi_{i}\left(\eta_{i}^{\rho} / \rho\right)^{\gamma-1}\right)=\frac{\Gamma(\gamma) \rho^{1-\alpha}}{\Gamma(\alpha)} \sum_{i=1}^{n} \xi_{i} \int_{0}^{\eta_{i}} \frac{\tau^{\rho-1} \Lambda_{\chi}(\tau)}{\left(\eta_{i}^{\rho}-\tau^{\rho}\right)^{1-\alpha}} d \tau .
$$

Thus,

$$
{ }_{\rho} \mathrm{I}_{0^{+}}^{1-\gamma} \chi(0)=\frac{\Gamma(\gamma) \rho^{1-\alpha}}{\Gamma(\alpha)} \Psi \sum_{i=1}^{n} \xi_{i} \int_{0}^{\eta_{i}} \frac{\tau^{\rho-1} \Lambda_{x}(\tau)}{\left(\eta_{i}^{\rho}-\tau^{\rho}\right)^{1-\alpha}} d \tau
$$

Therefore, by substitution of (2.5) in (2.3), we obtain that (2.1).

Secondly, we will prove the sufficient condition. By applying $\mathrm{I}_{0^{+}}^{1-\gamma}$ on both sides of the equation (2.1), we obtain

$$
\rho \mathrm{I}_{0^{+}}^{1-\gamma} \chi(\mathrm{t})={ }_{\rho} \mathrm{I}_{0^{+}}^{1-\gamma}\left(\mathrm{t}^{\rho} / \rho\right)^{\gamma-1} \frac{\Psi \rho^{1-\alpha}}{\Gamma(\alpha)} \sum_{i=1}^{n} \xi_{i} \int_{0}^{\eta_{i}} \frac{\tau^{\rho-1} \Lambda_{\chi}(\tau)}{\left(\eta_{i}^{\rho}-\tau^{\rho}\right)^{1-\alpha}} \mathrm{d} \tau+{ }_{\rho} \mathrm{I}_{0^{+}}^{1-\gamma}{ }_{\rho} \mathrm{I}_{0^{+}}^{\alpha} \Lambda_{\chi}(\mathrm{t}),
$$

Now, by using Lemmas 2.6 and 2.7, we have

$$
{ }_{\rho} \mathrm{I}_{0^{+}}^{1-\gamma} \chi(\mathrm{t})=\frac{\Gamma(\alpha) \rho^{1-\alpha}}{\Gamma(\alpha)} \Psi \sum_{i=1}^{n} \xi_{i} \int_{0}^{\eta_{i}} \frac{\tau^{\rho-1} \Lambda_{\chi}(\tau)}{\left(\eta_{i}^{\rho}-\tau^{\rho}\right)^{1-\alpha}} \mathrm{d} \tau+{ }_{\rho} \mathrm{I}_{0^{+}}^{1-\beta(1-\alpha)} \Lambda_{\chi}(\mathrm{t}) .
$$

Since $1-\beta(1-\alpha)>1-\gamma$, then by Lemma 2.9 and taking the limit as $t \rightarrow 0^{+}$yields

$$
{ }_{\rho} \mathrm{I}_{0^{+}}^{1-\gamma} \chi(0)=\frac{\Gamma(\gamma) \rho^{1-\alpha}}{\Gamma(\alpha)} \Psi \sum_{i=1}^{n} \xi_{i} \int_{0}^{\eta_{i}} \frac{\tau^{\rho-1} \Lambda_{\chi}(\tau)}{\left(\eta_{i}^{\rho}-\tau^{\rho}\right)^{1-\alpha}} d \tau
$$


Using the substitution $t=\eta_{i}$ in (2.1), we have

$$
x\left(\eta_{i}\right)=\frac{\Psi \rho^{1-\alpha}}{\Gamma(\alpha)}\left(\eta_{i}^{\rho} / \rho\right)^{\gamma-1} \sum_{i=1}^{n} \xi_{i} \int_{0}^{\eta_{i}} \frac{\tau^{\rho-1} \Lambda_{x}(\tau)}{\left(\eta_{i}^{\rho}-\tau^{\rho}\right)^{1-\alpha}} d \tau+\frac{\rho^{1-\alpha}}{\Gamma(\alpha)} \int_{0}^{\eta_{i}} \frac{\tau^{\rho-1} \Lambda_{x}(\tau)}{\left(\eta_{i}^{\rho}-\tau^{\rho}\right)^{1-\alpha}} d \tau .
$$

Furthermore, we derive

$$
\begin{aligned}
\sum_{i=1}^{n} \xi_{i} x\left(\eta_{i}\right)= & \frac{\Psi \rho^{1-\alpha}}{\Gamma(\alpha)}\left(\sum_{i=1}^{n} \xi_{i}\left(\eta_{i}^{\rho} / \rho\right)^{\gamma-1}\right) \sum_{i=1}^{n} \xi_{i} \int_{0}^{\eta_{i}} \frac{\tau^{\rho-1} \Lambda_{x}(\tau)}{\left(\eta_{i}^{\rho}-\tau^{\rho}\right)^{1-\alpha}} d \tau \\
& +\frac{\rho^{1-\alpha}}{\Gamma(\alpha)} \sum_{i=1}^{n} \xi_{i} \int_{0}^{\eta_{i}} \frac{\tau^{\rho-1} \Lambda_{x}(\tau)}{\left(\eta_{i}^{\rho}-\tau^{\rho}\right)^{1-\alpha}} d \tau \\
= & {\left[1+\Psi \sum_{i=1}^{n} \xi_{i}\left(\eta_{i}^{\rho} / \rho\right)^{\gamma-1}\right] \frac{\rho^{1-\alpha}}{\Gamma(\alpha)} \sum_{i=1}^{n} \xi_{i} \int_{0}^{\eta_{i}} \frac{\tau^{\rho-1} \Lambda_{x}(\tau)}{\left(\eta_{i}^{\rho}-\tau^{\rho}\right)^{1-\alpha}} d \tau } \\
= & \frac{\Gamma(\gamma) \rho^{1-\alpha}}{\Gamma(\alpha)} \Psi \sum_{i=1}^{n} \xi_{i} \int_{0}^{\eta_{i}} \frac{\tau^{\rho-1} \Lambda_{x}(\tau)}{\left(\eta_{i}^{\rho}-\tau^{\rho}\right)^{1-\alpha}} d \tau, \quad \text { (by (2.2)). }
\end{aligned}
$$

From equations (2.6) and (2.7), we obtain

$$
{ }_{\rho} \mathrm{I}_{0^{+}}^{1-\gamma} \chi(0)=\sum_{i=1}^{n} \xi_{i} \chi\left(\eta_{i}\right)
$$

Next, applying ${ }_{\rho} \mathrm{D}_{0^{+}}^{\gamma}$ on both sides of the equation (2.1) and by using Lemmas 2.6 and 2.10, we get

$$
{ }_{\rho} D_{0^{+}}^{\gamma} \chi(t)={ }_{\rho} D_{0^{+}}^{\beta(1-\alpha)} \Lambda_{x}(t)={ }_{\rho} D_{0^{+}}^{\beta(1-\alpha)} \varphi\left(t, x(t),{ }_{\rho} D_{0^{+}}^{\alpha, \beta} x(t)\right) .
$$

Since $x \in C_{1-\gamma, \rho}^{\gamma}[\mathbb{I}, \mathbb{R}]$ and by definition of $C_{1-\gamma, \rho}^{\gamma}[\mathbb{I}, \mathbb{R}]$ given in Definition 2.1, we have ${ }_{\rho} D_{0^{+}}^{\gamma} \chi(t) \in$ $C_{1-\gamma, \rho}[\mathbb{I}, \mathbb{R}]$ which implies that ${ }_{\rho} \mathrm{D}_{0^{+}}^{\beta(1-\alpha)} \varphi=\delta_{\rho}{ }_{\rho} \mathrm{I}_{0^{+}}^{1-\beta(1-\alpha)} \varphi \in \mathrm{C}_{1-\gamma, \rho}[\mathbb{I}, \mathbb{R}]$. For $\varphi \in \mathrm{C}_{1-\gamma, \rho}[\mathbb{I}, \mathbb{R}]$ it is clear that ${ }_{\rho} \mathrm{I}_{0^{+}}^{1-\beta(1-\alpha)} \varphi \in \mathrm{C}_{1-\gamma, \rho}[\mathbb{I}, \mathbb{R}]$. So, we have ${ }_{\rho} \mathrm{I}_{0^{+}}^{1-\beta(1-\alpha)} \varphi \in \mathrm{C}_{1-\gamma, \rho}^{1}[\mathbb{I}, \mathbb{R}]$. Therefore, $\varphi$ and ${ }_{\rho} \mathrm{I}_{0^{+}}^{1-\beta(1-\alpha)} \varphi$ satisfy conditions of Lemma 2.8. Applying ${ }_{\rho} \mathrm{I}_{0^{+}}^{\beta(1-\alpha)}$ on both sides of equation (2.8) and by using Lemma 2.8, we can get

$$
{ }_{\rho} D_{0^{+}}^{\alpha, \beta} x(t)=\Lambda_{x}(t)-\frac{\left({ }_{\rho} I_{0^{+}}^{1-\beta(1-\alpha)} \Lambda_{x}\right)(0)}{\Gamma(\beta(1-\alpha))}\left(t^{\rho} / \rho\right)^{\beta(1-\alpha)-1} .
$$

From Lemma 2.9, easily we can obtain that $\left.{ }_{\rho} \mathrm{I}_{0^{+}}^{1-\beta(1-\alpha)} \Lambda_{x}\right)(0)=0$. Thus, it reduces to ${ }_{\rho} \mathrm{D}_{0^{+}}^{\alpha, \beta} x(t)=$ $\Lambda_{\chi}(t)=\varphi\left(t, x(t), \rho_{\rho} D_{0^{+}}^{\alpha, \beta} \chi(t)\right)$.

\section{Main results}

In this section, we present the existence and uniqueness results of solutions for Hilfer-Katugampolatype fractional implicit differential equations given in the problem (1.2), then we discuss Ulam stability results for the Problem (1.2). For our main results, we need the following suppositions.

$\left(\mathcal{H}_{1}\right)$ Suppose that there exist $\phi, \psi, \chi \in C_{1-\gamma, \rho}[\mathbb{I}, \mathbb{R}]$ with $\phi^{*}=\sup _{t \in \mathbb{I}} \phi(t), \psi^{*}=\sup _{t \in \mathbb{I}} \psi(t)$ and $\chi^{*}=$ $\sup _{\mathrm{t} \in \mathbb{I}} \chi(\mathrm{t})<1$ such that

$$
|\varphi(t, p, q)| \leqslant \phi(t)+\psi(t)|p|+\chi(t)|q| \quad \text { for all } t \in \mathbb{I} ; p, q \in \mathbb{R}
$$


$\left(\mathcal{H}_{2}\right)$ Suppose that $\varphi \in C_{1-\gamma, \rho}^{\beta(1-\alpha)}[\mathbb{I}, \mathbb{R}]$ where $\varphi: \mathbb{I} \times \mathbb{R} \times \mathbb{R} \rightarrow \mathbb{R}$ be a function for any $\chi \in \mathrm{C}_{1-\gamma, \rho}^{\gamma}[\mathbb{I}, \mathbb{R}]$ and there exist $\mu>0$ and $\nu>0$ where $\mu, v$ are positive constants such that

$$
|\varphi(t, p, q)-\varphi(t, \tilde{p}, \tilde{q})| \leqslant \mu|p-\tilde{p}|+v|q-\tilde{q}| \quad \text { for any } p, q, \tilde{p}, \tilde{q} \in \mathbb{R} ; \quad t \in \mathbb{I} .
$$

$\left(\mathcal{H}_{3}\right)$ Suppose that the constant $\Theta$ satisfies the following estimate

$$
\Theta:=\left(\frac{\mu}{1-v}\right) \frac{B(\gamma, \alpha)}{\Gamma(\alpha)}\left[|\Psi| \sum_{i=1}^{n} \xi_{i}\left(\eta_{i}^{\rho} / \rho\right)^{\alpha+\gamma-1}+\left(T^{\rho} / \rho\right)^{\alpha}\right]<1 .
$$

$\left(\mathcal{H}_{4}\right)$ Suppose that there exist an increasing function $h \in \mathrm{C}_{1-\gamma, \mathrm{\rho}}[\mathbb{I}, \mathbb{R}]$ and $\zeta_{\mathrm{h}}>0$ such that

$$
{ }_{\rho} \mathrm{I}_{0^{+}}^{\alpha} h(t) \leqslant \zeta_{h} h(t), \quad \text { for any } t \in \mathbb{I} .
$$

Theorem 3.1 (Existence). Assume that $\left(\mathcal{H}_{1}\right)$ holds. Then, the problem $(1.2)$ has at least one solution in $\mathrm{C}_{1-\gamma, \rho}^{\gamma}[\mathbb{I}, \mathbb{R}]$ $\subset \mathrm{C}_{1-\gamma, \rho}^{\alpha, \beta}[\mathbb{I}, \mathbb{R}]$.

Proof. We will present the proof into several steps.

We consider the well defined operator $\mathcal{K}: C_{1-\gamma, \rho}[\mathbb{I}, \mathbb{R}] \rightarrow C_{1-\gamma, \rho}[\mathbb{I}, \mathbb{R}]$, which is given as follows

$$
(\mathcal{K} x)(t)=\frac{\Psi \rho^{1-\alpha}}{\Gamma(\alpha)}\left(t^{\rho} / \rho\right)^{\gamma-1} \sum_{i=1}^{n} \xi_{i} \int_{0}^{\eta_{i}} \frac{\tau^{\rho-1} \Lambda_{x}(\tau)}{\left(\eta_{i}^{\rho}-\tau^{\rho}\right)^{1-\alpha}} d \tau+\frac{\rho^{1-\alpha}}{\Gamma(\alpha)} \int_{0}^{t} \frac{\tau^{\rho-1} \Lambda_{\chi}(\tau)}{\left(t^{\rho}-\tau^{\rho}\right)^{1-\alpha}} d \tau .
$$

Step $1 . \mathcal{K}$ is continuous.

Assume that $x_{m}$ is a sequence such that $x_{m} \rightarrow x$ in $C_{1-\gamma, \rho}[\mathbb{I}, \mathbb{R}]$. Then, for each $t \in \mathbb{I}$, we have

$$
\begin{aligned}
\mid\left(\left(\mathcal{K} x_{m}\right)(t)\right. & -(\mathcal{K} x)(t))\left(t^{\rho} / \rho\right)^{1-\gamma} \mid \\
\leqslant & \frac{|\Psi| \rho^{1-\alpha}}{\Gamma(\alpha)} \sum_{i=1}^{n} \xi_{i} \int_{0}^{\eta_{i}} \frac{\tau^{\rho-1}}{\left(\eta_{i}^{\rho}-\tau^{\rho}\right)^{1-\alpha}}\left|\Lambda_{x_{m}}(\tau)-\Lambda_{x}(\tau)\right| d \tau \\
& +\frac{\left(t^{\rho} / \rho\right)^{1-\gamma} \rho^{1-\alpha}}{\Gamma(\alpha)} \int_{0}^{t} \frac{\tau^{\rho-1}}{\left(t^{\rho}-\tau^{\rho}\right)^{1-\alpha}}\left|\Lambda_{x_{m}}(\tau)-\Lambda_{x}(\tau)\right| d \tau \\
\leqslant & \frac{|\Psi| \rho^{1-\alpha}}{\Gamma(\alpha)} \sum_{i=1}^{n} \xi_{i} \int_{0}^{\eta_{i}} \frac{\tau^{\rho-1}\left(\tau^{\rho} / \rho\right)^{\gamma-1}}{\left(\eta_{i}^{\rho}-\tau^{\rho}\right)^{1-\alpha}} d \tau|| \Lambda_{x_{m}}(\cdot)-\Lambda_{x}(\cdot) \|_{C_{1-\gamma, \rho}} \\
& +\frac{\left(t^{\rho} / \rho\right)^{1-\gamma} \rho^{1-\alpha}}{\Gamma(\alpha)} \int_{0}^{t} \frac{\tau^{\rho-1}\left(\tau^{\rho} / \rho\right)^{\gamma-1}}{\left(t^{\rho}-\tau^{\rho}\right)^{1-\alpha}} \mathrm{d} \tau\left\|\Lambda_{x_{m}}(\cdot)-\Lambda_{x}(\cdot)\right\|_{C_{1-\gamma, \rho}} \\
\leqslant & \frac{B(\gamma, \alpha)}{\Gamma(\alpha)}|\Psi| \sum_{i=1}^{n} \xi_{i}\left(\eta_{i}^{\rho} / \rho\right)^{\alpha+\gamma-1}\left\|\Lambda_{x_{m}}(\cdot)-\Lambda_{x}(\cdot)\right\|_{C_{1-\gamma, \rho}} \\
& +\frac{B(\gamma, \alpha)}{\Gamma(\alpha)}\left(T^{\rho} / \rho\right)^{\alpha}\left\|\Lambda_{x_{m}}(\cdot)-\Lambda_{x}(\cdot)\right\|_{C_{1-\gamma, \rho}} \\
\leqslant & C\left\|\Lambda_{x_{m}}(\cdot)-\Lambda_{x}(\cdot)\right\|_{C_{1-\gamma, \rho}}
\end{aligned}
$$

where $C=\frac{B(\gamma, \alpha)}{\Gamma(\alpha)}\left[|\Psi| \sum_{i=1}^{n} \xi_{i}\left(\eta_{i}^{\rho} / \rho\right)^{\alpha+\gamma-1}+\left(T^{\rho} / \rho\right)^{\alpha}\right]$. Since $\Lambda_{x}$ is continuous (i.e. $\varphi$ is continuous), then (3.2) implies that

$$
\left\|\mathcal{K} x_{m}-\mathcal{K} x\right\|_{\mathrm{C}_{1-\gamma, \rho}} \rightarrow 0 \quad \text { as } \quad \mathrm{n} \rightarrow \infty
$$


Step 2. $\mathcal{K}$ maps bounded sets into bounded sets in $C_{1-\gamma, \rho}[\mathbb{I}, \mathbb{R}]$.

Actually, it is enough to show that for $\lambda>0$ there exists $\theta>0$ (positive constant) such that $x \in B_{\lambda}=$ $\left\{x \in C_{1-\gamma, \rho}[\mathbb{I}, \mathbb{R}]:\|x\|_{C_{1-\gamma, \rho}} \leqslant \lambda\right\}$, we have $\|\mathcal{K} x\|_{C_{1-\gamma, \rho}} \leqslant \theta$.

$$
\begin{aligned}
\left|((\mathcal{K} x)(\mathrm{t}))\left(\mathrm{t}^{\rho} / \rho\right)^{1-\gamma}\right| \leqslant & \frac{|\Psi| \rho^{1-\alpha}}{\Gamma(\alpha)} \sum_{i=1}^{n} \xi_{i} \int_{0}^{\eta_{i}} \frac{\tau^{\rho-1}}{\left(\eta_{i}^{\rho}-\tau^{\rho}\right)^{1-\alpha}}\left|\Lambda_{\chi}(\tau)\right| d \tau \\
& +\frac{\left(\mathrm{t}^{\rho} / \rho\right)^{1-\gamma} \rho^{1-\alpha}}{\Gamma(\alpha)} \int_{0}^{\mathrm{t}} \frac{\tau^{\rho-1}}{\left(\mathrm{t}^{\rho}-\tau^{\rho}\right)^{1-\alpha}}\left|\Lambda_{x}(\tau)\right| \mathrm{d} \tau .
\end{aligned}
$$

Here, we set

$$
\begin{aligned}
& \mathscr{A}=\frac{|\Psi| \rho^{1-\alpha}}{\Gamma(\alpha)} \sum_{i=1}^{n} \xi_{i} \int_{0}^{\eta_{i}} \frac{\tau^{\rho-1}}{\left(\eta_{i}^{\rho}-\tau^{\rho}\right)^{1-\alpha}}\left|\Lambda_{x}(\tau)\right| d \tau, \\
& \mathscr{B}=\frac{\left(t^{\rho} / \rho\right)^{1-\gamma} \rho^{1-\alpha}}{\Gamma(\alpha)} \int_{0}^{t} \frac{\tau^{\rho-1}}{\left(t^{\rho}-\tau^{\rho}\right)^{1-\alpha}}\left|\Lambda_{x}(\tau)\right| d \tau .
\end{aligned}
$$

Now, by supposition $\left(\mathcal{H}_{1}\right)$, we have

$$
\begin{aligned}
\left|\Lambda_{\chi}(t)\right|=\left|\varphi\left(t, \chi(t), \Lambda_{\chi}(t)\right)\right| & \leqslant \phi(t)+\psi(t)|\chi(t)|+\chi(t)\left|\Lambda_{\chi}(t)\right| \\
& \leqslant \phi^{*}+\psi^{*}|\chi(t)|+\chi^{*}\left|\Lambda_{\chi}(t)\right| \leqslant \frac{\phi^{*}+\psi^{*}|x(t)|}{1-\chi^{*}} .
\end{aligned}
$$

Thus, equations (3.4) and (3.5) become as follows:

$$
\begin{aligned}
& \mathscr{A}=\frac{|\Psi|}{1-\chi^{*}} \sum_{i=1}^{n} \xi_{i}\left[\frac{\phi^{*}\left(\eta_{i}^{\rho} / \rho\right)^{\alpha}}{\Gamma(1+\alpha)}+B(\gamma, \alpha) \frac{\psi^{*}\left(\eta_{i}^{\rho} / \rho\right)^{\alpha+\gamma-1}}{\Gamma(\alpha)}\|x\|_{C_{1-\gamma, \rho}}\right] \\
& \mathscr{B}=\frac{1}{1-\chi^{*}}\left[\frac{\phi^{*}\left(T^{\rho} / \rho\right)^{\alpha-\gamma+1}}{\Gamma(1+\alpha)}+B(\gamma, \alpha) \frac{\psi^{*}\left(T^{\rho} / \rho\right)^{\alpha}}{\Gamma(\alpha)}\|x\|_{C_{1-\gamma, \rho}}\right] .
\end{aligned}
$$

By putting equations (3.6) and (3.7) into (3.3), we obtain

$$
\begin{aligned}
\left|((\mathcal{K} x)(t))\left(t^{\rho} / \rho\right)^{1-\gamma}\right| \leqslant & \frac{\phi^{*}}{\left(1-\chi^{*}\right) \Gamma(1+\alpha)}\left[|\Psi| \sum_{i=1}^{n} \xi_{i}\left(\eta_{i}^{\rho} / \rho\right)^{\alpha}+\left(T^{\rho} / \rho\right)^{\alpha-\gamma+1}\right] \\
& +\frac{\psi^{*} B(\gamma, \alpha)}{\left(1-\chi^{*}\right) \Gamma(\alpha)}\left[|\Psi| \sum_{i=1}^{n} \xi_{i}\left(\eta_{i}^{\rho} / \rho\right)^{\alpha+\gamma-1}+\left(T^{\rho} / \rho\right)^{\alpha}\right]\|x\|_{C_{1-\gamma, \rho}}:=\theta
\end{aligned}
$$

Step: 3. $\mathcal{K}$ maps bounded sets into equicontinuous set of $C_{1-\gamma, \rho}[\mathbb{I}, \mathbb{R}]$.

Assume that $t_{2} \leqslant t_{1}$ for $t_{2}, t_{1} \in \mathbb{I}, B_{\lambda}$ is the bounded set of $C_{1-\gamma, \rho}[\mathbb{I}, \mathbb{R}]$ as in the Step 2 , and $x \in B_{\lambda}$. Then, we have

$$
\begin{aligned}
& \left|\left((\mathcal{K} x)\left(\mathrm{t}_{1}\right)\right)\left(\mathrm{t}_{1}^{\rho} / \rho\right)^{1-\gamma}-\left((\mathcal{K} x)\left(\mathrm{t}_{2}\right)\right)\left(\mathrm{t}_{2}^{\rho} / \rho\right)^{1-\gamma}\right| \\
& \leqslant\left|\frac{\left(\mathrm{t}_{1}^{\rho} / \rho\right)^{1-\gamma} \rho^{1-\alpha}}{\Gamma(\alpha)} \int_{0}^{\mathrm{t}_{1}} \frac{\tau^{\rho-1} \Lambda_{x}(\tau)}{\left(\mathrm{t}_{1}^{\rho}-\tau^{\rho}\right)^{1-\alpha}} \mathrm{d} \tau-\frac{\left(\mathrm{t}_{2}^{\rho} / \rho\right)^{1-\gamma} \rho^{1-\alpha}}{\Gamma(\alpha)} \int_{0}^{\mathrm{t}_{2}} \frac{\tau^{\rho-1} \Lambda_{x}(\tau)}{\left(\mathrm{t}_{2}^{\rho}-\tau^{\rho}\right)^{1-\alpha}} \mathrm{d} \tau\right| \\
& \leqslant\left|\frac{\rho^{1-\alpha}}{\Gamma(\alpha)} \int_{0}^{\mathrm{t}_{1}}\left[\frac{\left(\mathrm{t}_{1}^{\rho} / \rho\right)^{1-\gamma}}{\left(\mathrm{t}_{1}^{\rho}-\tau^{\rho}\right)^{1-\alpha}}-\frac{\left(\mathrm{t}_{2}^{\rho} / \rho\right)^{1-\gamma}}{\left(\mathrm{t}_{2}^{\rho}-\tau^{\rho}\right)^{1-\alpha}}\right] \tau^{\rho-1} \Lambda_{x}(\tau) \mathrm{d} \tau\right| \\
& \quad+\left|\frac{\left(\mathrm{t}_{2}^{\rho} / \rho\right)^{1-\gamma} \rho^{1-\alpha}}{\Gamma(\alpha)} \int_{\mathrm{t}_{1}}^{\mathrm{t}_{2}} \frac{\tau^{\rho-1} \Lambda_{x}(\tau)}{\left(\mathrm{t}_{2}^{\rho}-\tau^{\rho}\right)^{1-\alpha}} \mathrm{d} \tau\right| .
\end{aligned}
$$


The right-hand side of the inequality (3.8) tends to zero, as $t_{1} \rightarrow t_{2}$.

Consequently, from Steps 1-3, together with Arzela-Ascoli theorem, we can deduce that

$$
\mathcal{K}: \mathrm{C}_{1-\gamma, \rho}[\mathbb{I}, \mathbb{R}] \rightarrow \mathrm{C}_{1-\gamma, \rho}[\mathbb{I}, \mathbb{R}]
$$

is a completely continuous.

Final Step. We will show that the set

$$
\mathrm{G}=\left\{x \in \mathrm{C}_{1-\gamma, \rho}[\mathbb{I}, \mathbb{R}]: x=\varepsilon(\mathcal{K} x), \quad 0 \leqslant \varepsilon \leqslant 1\right\}
$$

is a bounded set.

Assume that $x \in G, x=\varepsilon(\mathcal{K} x)$ for some $0 \leqslant \varepsilon \leqslant 1$. Then, for each $t \in \mathbb{I}$, we have

$$
x(t)=\varepsilon\left(\frac{|\Psi| \rho^{1-\alpha}}{\Gamma(\alpha)} \sum_{i=1}^{n} \xi_{i} \int_{0}^{\eta_{i}} \frac{\tau^{\rho-1} \Lambda_{x}(\tau)}{\left(\eta_{i}^{\rho}-\tau^{\rho}\right)^{1-\alpha}} d \tau+\frac{\left(t^{\rho} / \rho\right)^{1-\gamma} \rho^{1-\alpha}}{\Gamma(\alpha)} \int_{0}^{t} \frac{\tau^{\rho-1} \Lambda_{x}(\tau)}{\left(t^{\rho}-\tau^{\rho}\right)^{1-\alpha}} d \tau\right) .
$$

Hence, by supposition $\left(\mathcal{H}_{1}\right)$, the equation (3.9) implies that

$$
\begin{aligned}
\left|(x(t))\left(t^{\rho} / \rho\right)^{1-\gamma}\right| \leqslant & \left|((\mathcal{K} x)(t))\left(t^{\rho} / \rho\right)^{1-\gamma}\right| \\
\leqslant & \frac{\phi^{*}}{\left(1-\chi^{*}\right) \Gamma(1+\alpha)}\left[|\Psi| \sum_{i=1}^{n} \xi_{i}\left(\eta_{i}^{\rho} / \rho\right)^{\alpha}+\left(T^{\rho} / \rho\right)^{\alpha-\gamma+1}\right] \\
& +\frac{\psi^{*} B(\gamma, \alpha)}{\left(1-\chi^{*}\right) \Gamma(\alpha)}\left[|\Psi| \sum_{i=1}^{n} \xi_{i}\left(\eta_{i}^{\rho} / \rho\right)^{\alpha+\gamma-1}+\left(T^{\rho} / \rho\right)^{\alpha}\right]\|x\|_{C_{1-\gamma, \rho}}:=\theta_{1} .
\end{aligned}
$$

Therefore, the set $\mathrm{G}$ is a bounded. Consequently, by Lemma 2.4 (Schaefer's fixed point theorem), we conclude that $\mathcal{K}$ has a fixed point which is a solution of problem (1.2).

Theorem 3.2 (Uniqueness). Assume that $\left(\mathcal{H}_{2}\right)$ and $\left(\mathcal{H}_{3}\right)$ are satisfied. Then, the problem (1.2) has a unique solution.

Proof. Assume that the operator $\mathcal{K}: C_{1-\gamma, \rho}[\mathbb{I}, \mathbb{R}] \rightarrow C_{1-\gamma, \rho}[\mathbb{I}, \mathbb{R}]$, defined in equation (3.1). Clearly, by Lemma 2.11, the fixed points of the operator $\mathcal{K}$ are solutions of the problem (1.2). Now, it remains to show that the solution is unique. Suppose that $x_{1}, x_{2} \in C_{1-\gamma, \rho}[\mathbb{I}, \mathbb{R}]$ and $t \in \mathbb{I}$, then we have

$$
\begin{aligned}
\left|\left(\left(\mathcal{K} x_{1}\right)(t)-\left(\mathcal{K} x_{2}\right)(t)\right)\left(t^{\rho} / \rho\right)^{1-\gamma}\right| \leqslant & \frac{|\Psi| \rho^{1-\alpha}}{\Gamma(\alpha)} \sum_{i=1}^{n} \xi_{i} \int_{0}^{\eta_{i}} \frac{\tau^{\rho-1}}{\left(\eta_{i}^{\rho}-\tau^{\rho}\right)^{1-\alpha}}\left|\Lambda_{x_{1}}(\tau)-\Lambda_{x_{2}}(\tau)\right| d \tau \\
& +\frac{\left(t^{\rho} / \rho\right)^{1-\gamma} \rho^{1-\alpha}}{\Gamma(\alpha)} \int_{0}^{t} \frac{\tau^{\rho-1}}{\left(t^{\rho}-\tau^{\rho}\right)^{1-\alpha}}\left|\Lambda_{x_{1}}(\tau)-\Lambda_{x_{2}}(\tau)\right| d \tau
\end{aligned}
$$

By supposition $\left(\mathcal{H}_{2}\right)$, we have

$$
\begin{aligned}
\left|\Lambda_{x_{1}}(t)-\Lambda_{x_{2}}(t)\right| & =\left|\varphi\left(t, x_{1}(t), \Lambda_{x_{1}}(t)\right)-\varphi\left(t, x_{2}(t), \Lambda_{x_{2}}(t)\right)\right| \\
& \leqslant \mu\left|x_{1}(t)-x_{2}(t)\right|+v\left|\Lambda_{x_{1}}(t)-\Lambda_{x_{2}}(t)\right| \leqslant \frac{\mu}{1-v}\left|x_{1}(t)-x_{2}(t)\right| .
\end{aligned}
$$

By substituting (3.11) in the inequality (3.10), we obtain

$$
\begin{aligned}
\left|\left(\left(\mathcal{K} x_{1}\right)(t)-\left(\mathcal{K} x_{2}\right)(t)\right)\left(t^{\rho} / \rho\right)^{1-\gamma}\right| \leqslant & \frac{B(\gamma, \alpha)}{\Gamma(\alpha)}\left(\frac{\mu|\Psi|}{1-v}\right) \sum_{i=1}^{n} \xi_{i}\left(\eta_{i}^{\rho} / \rho\right)^{\alpha+\gamma-1}\left\|x_{1}-x_{1}\right\|_{C_{1-\gamma, \rho}} \\
& +\frac{B(\gamma, \alpha)}{\Gamma(\alpha)}\left(\frac{\mu}{1-v}\right)\left(T^{\rho} / \rho\right)^{\alpha}\left\|x_{1}-x_{1}\right\|_{C_{1-\gamma, \rho}} \leqslant \Theta\left\|x_{1}-x_{1}\right\|_{C_{1-\gamma, \rho}} .
\end{aligned}
$$


Thus, $\left\|\left(\mathcal{K} x_{1}\right)(t)-\left(\mathcal{K} x_{2}\right)(t)\right\|_{C_{1-\gamma, \rho}} \leqslant \Theta\left\|x_{1}-x_{1}\right\|_{C_{1-\gamma, \rho}}$. It follows from supposition $\left(\mathcal{H}_{3}\right)$, that the operator $\mathcal{K}$ is contraction. By well known Banach contraction principle, we can deduce that $\mathcal{K}$ has a unique fixed point which is a solution of the problem (1.2).

Next, we will prove some different types of Ulam stability results for the fractional implicit differential equations (1.2). Now, we shall give definitions and criteria of Ulam-Hyers stable (U.H.S.) and Ulam-HyersRassias stable (U.H.R.S.) for the Hilfer-Katugampola fractional implicit differential equations (1.2).

For $\delta>0$ and $h: \mathbb{I} \rightarrow[0, \infty)$ is a continuous function, we theorize the following inequalities

$$
\begin{aligned}
& \left|{ }_{\rho} D_{0^{+}}^{\alpha, \beta} y(t)-\varphi\left(t, y(t),{ }_{\rho} D_{0^{+}}^{\alpha, \beta} y(t)\right)\right| \leqslant \delta, \quad t \in \mathbb{I} \\
& \left|{ }_{\rho} D_{0^{+}}^{\alpha, \beta} y(t)-\varphi\left(t, y(t),{ }_{\rho} D_{0^{+}}^{\alpha, \beta} y(t)\right)\right| \leqslant \delta h(t), \quad t \in \mathbb{I} \\
& \left|{ }_{\rho} D_{0^{+}}^{\alpha, \beta} y(t)-\varphi\left(t, y(t),{ }_{\rho} D_{0^{+}}^{\alpha, \beta} y(t)\right)\right| \leqslant h(t), \quad t \in \mathbb{I} .
\end{aligned}
$$

Definition 3.3. The problem (1.2) is U.H.S. if there exists the real number $\lambda_{\varphi}>0$ such that for all $\delta>0$ and for all solution $y \in C_{1-\gamma, \rho}^{\gamma}[\mathbb{I}, \mathbb{R}]$ of the inequality (3.12) there exists the solution $x \in C_{1-\gamma, \rho}^{\gamma}[\mathbb{I}, \mathbb{R}]$ of the problem (1.2) satisfying

$$
|y(t)-x(t)| \leqslant \lambda_{\varphi} \delta, \quad t \in \mathbb{I} .
$$

Definition 3.4. The problem (1.2) is generalized U.H.S. if there exists $z_{\varphi} \in C([0, \infty),[0, \infty)), z_{\varphi}(0)=0$ such that for all solution $y \in \mathrm{C}_{1-\gamma, \rho}^{\gamma}[\mathbb{I}, \mathbb{R}]$ of the inequality (3.12) there exists the solution $x \in \mathrm{C}_{1-\gamma, \rho}^{\gamma}[\mathbb{I}, \mathbb{R}]$ of the problem (1.2) satisfying

$$
|y(t)-x(t)| \leqslant z_{\varphi} \delta, \quad t \in \mathbb{I} .
$$

Definition 3.5. The problem (1.2) is U.H.R.S. with respect to $h \in C_{1-\gamma, \rho}[\mathbb{I}, \mathbb{R}]$ if there exists the real number $\lambda_{\varphi}>0$ such that for all $\delta>0$ and for all solution $y \in C_{1-\gamma, \rho}^{\gamma}[\mathbb{I}, \mathbb{R}]$ of the inequality (3.13) there exists the solution $x \in \mathrm{C}_{1-\gamma, \rho}^{\gamma}[\mathrm{I}, \mathbb{R}]$ of the problem (1.2) satisfying

$$
|y(t)-x(t)| \leqslant \lambda_{\varphi} \delta h(t), \quad t \in \mathbb{I}
$$

Definition 3.6. The problem (1.2) is generalized U.H.R.S. with respect to $h \in C_{1-\gamma, \rho}[\mathbb{I}, \mathbb{R}]$ if there exists the real number $\lambda_{\varphi, h}>0$ such that for all solution $y \in C_{1-\gamma, \rho}^{\gamma}[\mathbb{I}, \mathbb{R}]$ of the inequality (3.14) there exists the solution $x \in \mathrm{C}_{1-\gamma, \rho}^{\gamma}[\mathbb{I}, \mathbb{R}]$ of the problem (1.2) satisfying

$$
|y(t)-x(t)| \leqslant \lambda_{\varphi, h} h(t), \quad t \in \mathbb{I} .
$$

Remark 3.7. From the above definitions it is clear that

1. Definition $3.3 \Rightarrow$ Definition 3.4;

2. Definition $3.5 \Rightarrow$ Definition 3.6;

3. Definition 3.5 for $h(\cdot)=1 \Rightarrow$ Definition 3.3.

Remark 3.8. The function $y \in \mathrm{C}_{1-\gamma, \rho}^{\gamma}[\mathrm{I}, \mathbb{R}]$ is the solution of the inequality (3.12), if and only if there exists the function $z \in \mathrm{C}_{1-\gamma, \rho}^{\gamma}[\mathbb{I}, \mathbb{R}]$ such that

(a) $|z(t)| \leqslant \delta, \quad t \in \mathbb{I} ;$

(b) ${ }_{\rho} \mathrm{D}_{0^{+}}^{\alpha, \beta} \mathrm{y}(\mathrm{t})=\varphi\left(t, y(t),{ }_{\rho} \mathrm{D}_{0^{+}}^{\alpha, \beta} \mathrm{y}(\mathrm{t})\right)+z(\mathrm{t}), \quad \mathrm{t} \in \mathbb{I}$.

We have similar remarks for the inequalities (3.13) and (3.14). 
Lemma 3.9. Assume that $\rho>0,0<\alpha<1$, and $\beta \in[0,1]$. If the function $y \in \mathrm{C}_{1-\gamma, \rho}^{\gamma}[\mathbb{I}, \mathbb{R}]$ is the solution of the inequality (3.12), then $y$ is the solution of the following integral inequality

$$
\left|y(t)-\Xi_{y}-\frac{\rho^{1-\alpha}}{\Gamma(\alpha)} \int_{0}^{t} \frac{\tau^{\rho-1} \Lambda_{y}(\tau)}{\left(t^{\rho}-\tau^{\rho}\right)^{1-\alpha}} d \tau\right| \leqslant\left[|\Psi|(n \xi)\left(T^{\rho} / \rho\right)^{\alpha+\gamma-1}+\left(T^{\rho} / \rho\right)^{\alpha}\right] \frac{\delta}{\Gamma(1+\alpha)^{\prime}},
$$

where,

$$
\Xi_{y}=\frac{\Psi \rho^{1-\alpha}}{\Gamma(\alpha)}\left(t^{\rho} / \rho\right)^{\gamma-1} \sum_{i=1}^{n} \xi_{i} \int_{0}^{\eta_{i}} \frac{\tau^{\rho-1} \Lambda_{y}(\tau)}{\left(\eta_{i}^{\rho}-\tau^{\rho}\right)^{1-\alpha}} d \tau, \quad \xi=\max _{i=1,2, \ldots, n}\left\{\xi_{i}\right\} .
$$

Proof. In view of Remark 3.8, we have

$$
{ }_{\rho} D_{0^{+}}^{\alpha, \beta} y(t)=\varphi\left(t, y(t),{ }_{\rho} D_{0^{+}}^{\alpha, \beta} y(t)\right)+z(t)=\Lambda_{y}(t)+z(t) .
$$

Hence,

$$
\begin{aligned}
y(t)= & \frac{\Psi \rho^{1-\alpha}}{\Gamma(\alpha)}\left(t^{\rho} / \rho\right)^{\gamma-1} \sum_{i=1}^{n} \xi_{i}\left[\int_{0}^{\eta_{i}} \frac{\tau^{\rho-1} \Lambda_{y}(\tau)}{\left(\eta_{i}^{\rho}-\tau^{\rho}\right)^{1-\alpha}} d \tau+\int_{0}^{\eta_{i}} \frac{\tau^{\rho-1} z(\tau)}{\left(\eta_{i}^{\rho}-\tau^{\rho}\right)^{1-\alpha}} d \tau\right] \\
& +\frac{\rho^{1-\alpha}}{\Gamma(\alpha)}\left[\int_{0}^{t} \frac{\tau^{\rho-1} \Lambda_{y}(\tau)}{\left(t^{\rho}-\tau^{\rho}\right)^{1-\alpha}} d \tau+\int_{0}^{t} \frac{\tau^{\rho-1} z(\tau)}{\left(t^{\rho}-\tau^{\rho}\right)^{1-\alpha}} d \tau\right] .
\end{aligned}
$$

It follows from (3.15) and (3.16) that

$$
\begin{aligned}
\mid y(t) & -\Xi_{y}-\frac{\rho^{1-\alpha}}{\Gamma(\alpha)} \int_{0}^{t} \frac{\tau^{\rho-1} \Lambda_{y}(\tau)}{\left(t^{\rho}-\tau^{\rho}\right)^{1-\alpha}} d \tau \mid \\
& =\left|\frac{\Psi \rho^{1-\alpha}}{\Gamma(\alpha)}\left(t^{\rho} / \rho\right)^{\gamma-1} \sum_{i=1}^{n} \xi_{i} \int_{0}^{\eta_{i}} \frac{\tau^{\rho-1} z(\tau)}{\left(\eta_{i}^{\rho}-\tau^{\rho}\right)^{1-\alpha}} d \tau+\frac{\rho^{1-\alpha}}{\Gamma(\alpha)} \int_{0}^{t} \frac{\tau^{\rho-1} z(\tau)}{\left(t^{\rho}-\tau^{\rho}\right)^{1-\alpha}} d \tau\right| \\
& \leqslant \frac{|\Psi| \rho^{1-\alpha}}{\Gamma(\alpha)}\left(t^{\rho} / \rho\right)^{\gamma-1} \sum_{i=1}^{n} \xi_{i} \int_{0}^{\eta_{i}} \frac{\tau^{\rho-1}}{\left(\eta_{i}^{\rho}-\tau^{\rho}\right)^{1-\alpha}}|z(\tau)| d \tau+\frac{\rho^{1-\alpha}}{\Gamma(\alpha)} \int_{0}^{t} \frac{\tau^{\rho-1}}{\left(t^{\rho}-\tau^{\rho}\right)^{1-\alpha}}|z(\tau)| d \tau \\
& \leqslant \frac{|\Psi| \rho^{1-\alpha}}{\Gamma(\alpha)}\left(t^{\rho} / \rho\right)^{\gamma-1} \sum_{i=1}^{n} \xi_{i} \int_{0}^{\eta_{i}} \frac{\tau^{\rho-1} \delta}{\left(\eta_{i}^{\rho}-\tau^{\rho}\right)^{1-\alpha}} d \tau+\frac{\rho^{1-\alpha}}{\Gamma(\alpha)} \int_{0}^{t} \frac{\tau^{\rho-1} \delta}{\left(t^{\rho}-\tau^{\rho}\right)^{1-\alpha}} d \tau \\
& \leqslant\left[|\Psi|(n \xi)\left(T^{\rho} / \rho\right)^{\alpha+\gamma-1}+\left(T^{\rho} / \rho\right)^{\alpha}\right] \frac{\delta}{\Gamma(1+\alpha)} .
\end{aligned}
$$

Lemma 3.10. Assume that $\rho>0,0<\alpha<1$, and $\beta \in[0,1]$. If the function $y \in C_{1-\gamma, \rho}^{\gamma}[\mathbb{I}, \mathbb{R}]$ is the solution of the inequality (3.13), then $y$ is the solution of the following integral inequality

$$
\left|y(t)-\Xi_{y}-\frac{\rho^{1-\alpha}}{\Gamma(\alpha)} \int_{0}^{t} \frac{\tau^{\rho-1} \Lambda_{y}(\tau)}{\left(t^{\rho}-\tau^{\rho}\right)^{1-\alpha}} d \tau\right| \leqslant\left[|\Psi|(n \xi)\left(t^{\rho} / \rho\right)^{\gamma-1}+1\right] \delta \zeta_{h} h(t),
$$

where,

$$
\Xi_{y}=\frac{\Psi \rho^{1-\alpha}}{\Gamma(\alpha)}\left(t^{\rho} / \rho\right)^{\gamma-1} \sum_{i=1}^{n} \xi_{i} \int_{0}^{\eta_{i}} \frac{\tau^{\rho-1} \Lambda_{y}(\tau)}{\left(\eta_{i}^{\rho}-\tau^{\rho}\right)^{1-\alpha}} d \tau, \quad \xi=\max _{i=1,2, \ldots, n}\left\{\xi_{i}\right\},
$$

and $\zeta_{\mathrm{h}}$ is a positive constant.

Proof. Proof of this Lemma is directly follows from Remark 3.8 and Lemma 3.9 with the help of supposition $\left(\mathcal{H}_{4}\right)$.

Notice that, we can find a similar remark for the inequalities (3.14).

Now, We will state a generalization of Gronwall's lemma for singular kernels which we are using to discuss our stability results for the problem (1.2). 
Lemma 3.11 ([34]). Assume that $\mathrm{f}:[0, \mathrm{~T}] \rightarrow[0, \infty)$ is a real function and $\mathrm{g}(\cdot)$ is a non-negative locally integrable function on $[0, T]$. And let there exists $\rho>0, \omega>0$, and $0<\alpha<1$, such that

$$
f(t) \leqslant g(t)+\omega \int_{0}^{t} \tau^{\rho-1} \frac{\rho^{\alpha}}{\left(t^{\rho}-\tau^{\rho}\right)^{\alpha}} f(\tau) d \tau .
$$

Then, there exists a constant $\mathrm{C}^{*}=\mathrm{C}^{*}(\alpha)$ such that for $\mathrm{t} \in[0, \mathrm{~T}]$, we have

$$
f(t) \leqslant g(t)+C^{*} \omega \int_{0}^{t} \tau^{\rho-1} \frac{\rho^{\alpha}}{\left(t^{\rho}-\tau^{\rho}\right)^{\alpha}} g(\tau) d \tau .
$$

Theorem 3.12. Assume that the hypotheses $\left(\mathcal{H}_{2}\right)$ and $\left(\mathcal{H}_{3}\right)$ are satisfied. Then, the problem (1.2) is U.H.S..

Proof. Let $\delta>0$ and let for any $t \in \mathbb{I}$ the function $y \in C_{1-\gamma, \rho}^{\gamma}[\mathbb{I}, \mathbb{R}]$ satisfies the inequality (3.12). In the light of Theorem 3.2, $x \in \mathrm{C}_{1-\gamma, \rho}^{\gamma}[\mathbb{I}, \mathbb{R}]$ is a unique solution of implicit fractional differential equation satisfying

$$
\begin{cases}{ }_{\rho} \mathrm{D}_{0^{+}}^{\alpha, \beta} \chi(t)=\varphi\left(t, \chi(t),{ }_{\rho} \mathrm{D}_{0^{+}}^{\alpha, \beta} \chi(t)\right), & t \in \mathbb{I}:=[0, T], 0<\alpha<1,0 \leqslant \beta \leqslant 1, \\ { }_{\rho} \mathrm{I}_{0^{+}}^{1-\gamma} \chi(0)={ }_{\rho} \mathrm{I}_{0^{+}}^{1-\gamma} \mathrm{y}(0)=\sum_{i=1}^{n} \xi_{i} \chi\left(\eta_{i}\right), & \gamma=\alpha+\beta(1-\alpha) .\end{cases}
$$

By using Lemma 2.11, we get

$$
x(t)=\Xi_{x}+\frac{\rho^{1-\alpha}}{\Gamma(\alpha)} \int_{0}^{t} \frac{\tau^{\rho-1} \Lambda_{x}(\tau)}{\left(t^{\rho}-\tau^{\rho}\right)^{1-\alpha}} d \tau
$$

where

$$
\Xi_{x}=\frac{\Psi \rho^{1-\alpha}}{\Gamma(\alpha)}\left(t^{\rho} / \rho\right)^{\gamma-1} \sum_{i=1}^{n} \xi_{i} \int_{0}^{\eta_{i}} \frac{\tau^{\rho-1} \Lambda_{x}(\tau)}{\left(\eta_{i}^{\rho}-\tau^{\rho}\right)^{1-\alpha}} d \tau
$$

Now, if $x\left(\eta_{i}\right)=y\left(\eta_{i}\right)$ and ${ }_{\rho} I_{0^{+}}^{1-\gamma} x(0)={ }_{\rho} I_{0^{+}}^{1-\gamma} y(0)$, then $\Xi_{x}=\Xi_{y}$. Clearly,

$$
\begin{aligned}
\left|\Xi_{x}-\Xi_{y}\right| & \leqslant \frac{|\Psi| \rho^{1-\alpha}}{\Gamma(\alpha)}\left(t^{\rho} / \rho\right)^{\gamma-1} \sum_{i=1}^{n} \xi_{i} \int_{0}^{\eta_{i}} \frac{\tau^{\rho-1}}{\left(\eta_{i}^{\rho}-\tau^{\rho}\right)^{1-\alpha}}\left|\Lambda_{x}(\tau)-\Lambda_{y}(\tau)\right| d \tau \\
& \leqslant \frac{|\Psi| \rho^{1-\alpha}}{\Gamma(\alpha)}\left(t^{\rho} / \rho\right)^{\gamma-1} \sum_{i=1}^{n} \xi_{i} \int_{0}^{\eta_{i}} \frac{\tau^{\rho-1}}{\left(\eta_{i}^{\rho}-\tau^{\rho}\right)^{1-\alpha}}\left(\frac{\mu}{1-v}\right)|x(\tau)-y(\tau)| d \tau \\
& \leqslant\left(\frac{\mu}{1-v}\right) \frac{|\Psi| \rho^{1-\alpha}}{\Gamma(\alpha)}\left(t^{\rho} / \rho\right)^{\gamma-1} \sum_{i=1}^{n} \xi_{i} I_{0^{+}}^{\alpha}\left|x\left(\eta_{i}\right)-y\left(\eta_{i}\right)\right|=0 .
\end{aligned}
$$

Thus, we have

$$
x(t)=\Xi_{y}+\frac{\rho^{1-\alpha}}{\Gamma(\alpha)} \int_{0}^{t} \frac{\tau^{\rho-1} \Lambda_{x}(\tau)}{\left(t^{\rho}-\tau^{\rho}\right)^{1-\alpha}} d \tau .
$$

By applying Lemma 3.9 and integration of inequality (3.12) for any $t \in \mathbb{I}$, we have

$$
\begin{aligned}
|y(t)-x(t)| \leqslant\left|y(t)-\Xi_{y}-\frac{\rho^{1-\alpha}}{\Gamma(\alpha)} \int_{0}^{t} \frac{\tau^{\rho-1} \Lambda_{y}(\tau)}{\left(t^{\rho}-\tau^{\rho}\right)^{1-\alpha}} d \tau\right|+\frac{\rho^{1-\alpha}}{\Gamma(\alpha)} \int_{0}^{t} \frac{\tau^{\rho-1}}{\left(t^{\rho}-\tau^{\rho}\right)^{1-\alpha}}\left|\Lambda_{y}(\tau)-\Lambda_{x}(\tau)\right| d \tau \\
\leqslant\left|y(t)-\Xi_{y}-\frac{\rho^{1-\alpha}}{\Gamma(\alpha)} \int_{0}^{t} \frac{\tau^{\rho-1} \Lambda_{y}(\tau)}{\left(t^{\rho}-\tau^{\rho}\right)^{1-\alpha}} d \tau\right| \\
\quad+\left(\frac{\mu}{1-v}\right) \frac{\rho^{1-\alpha}}{\Gamma(\alpha)} \int_{0}^{t} \frac{\tau^{\rho-1}}{\left(t^{\rho}-\tau^{\rho}\right)^{1-\alpha}}|y(\tau)-x(\tau)| d \tau .
\end{aligned}
$$

By the inequality (3.15), we can write the inequality (3.19) as follows

$$
\begin{aligned}
|y(t)-x(t)| \leqslant & {\left[|\Psi|(n \xi)\left(T^{\rho} / \rho\right)^{\alpha+\gamma-1}+\left(T^{\rho} / \rho\right)^{\alpha}\right] \frac{\delta}{\Gamma(1+\alpha)} } \\
& +\left(\frac{\mu}{1-v}\right) \frac{\rho^{1-\alpha}}{\Gamma(\alpha)} \int_{0}^{t} \frac{\tau^{\rho-1}}{\left(t^{\rho}-\tau^{\rho}\right)^{1-\alpha}}|y(\tau)-x(\tau)| d \tau .
\end{aligned}
$$


Now, applying Lemma 3.11, we get

$$
|y(t)-x(t)| \leqslant\left[|\Psi|(n \xi)\left(T^{\rho} / \rho\right)^{\alpha+\gamma-1}+\left(T^{\rho} / \rho\right)^{\alpha}\right]\left(1+\frac{C_{1} \mu}{(1-v) \Gamma(\alpha)}\left(T^{\rho} / \rho\right)^{\alpha}\right) \frac{\delta}{\Gamma(1+\alpha)}:=\lambda_{\varphi} \delta,
$$

where $C_{1}=C_{1}(\alpha)=\frac{1}{\alpha}$ be a constant. Furthermore, if we put $z(\delta)=\lambda_{\varphi} \delta ; z(0)=0$, then the problem (1.2) is generalized U.H.S..

Theorem 3.13. Suppose that the hypotheses $\left(\mathcal{H}_{2}\right),\left(\mathcal{H}_{3}\right)$, and $\left(\mathcal{H}_{4}\right)$ are satisfied. Then, the problem (1.2) is U.H.R.S.

Proof. Let $\delta>0$ and let for any $t \in \mathbb{I}$ the function $y \in C_{1-\gamma, \rho}^{\gamma}[\mathbb{I}, \mathbb{R}]$ satisfies the inequality (3.13). In the light of Theorem 3.2, $x \in \mathrm{C}_{1-\gamma, \rho}^{\gamma}[\mathbb{I}, \mathbb{R}]$ is a unique solution of implicit fractional differential equation satisfying the problem (3.18).

By using same arguments in the above theorem, we obtain

$$
x(t)=\Xi_{y}+\frac{\rho^{1-\alpha}}{\Gamma(\alpha)} \int_{0}^{t} \frac{\tau^{\rho-1} \Lambda_{x}(\tau)}{\left(t^{\rho}-\tau^{\rho}\right)^{1-\alpha}} d \tau
$$

where

$$
\Xi_{y}=\frac{\Psi \rho^{1-\alpha}}{\Gamma(\alpha)}\left(t^{\rho} / \rho\right)^{\gamma-1} \sum_{i=1}^{n} \xi_{i} \int_{0}^{\eta_{i}} \frac{\tau^{\rho-1} \Lambda_{y}(\tau)}{\left(\eta_{i}^{\rho}-\tau^{\rho}\right)^{1-\alpha}} d \tau .
$$

Now, applying Lemma 3.10 and integration of inequality (3.13) for any $t \in \mathbb{I}$, we have

$$
\begin{aligned}
|y(t)-x(t)| \leqslant & \left|y(t)-\Xi_{y}-\frac{\rho^{1-\alpha}}{\Gamma(\alpha)} \int_{0}^{t} \frac{\tau^{\rho-1} \Lambda_{y}(\tau)}{\left(t^{\rho}-\tau^{\rho}\right)^{1-\alpha}} d \tau\right| \\
& +\left(\frac{\mu}{1-v}\right) \frac{\rho^{1-\alpha}}{\Gamma(\alpha)} \int_{0}^{t} \frac{\tau^{\rho-1}}{\left(t^{\rho}-\tau^{\rho}\right)^{1-\alpha}}|y(\tau)-x(\tau)| d \tau .
\end{aligned}
$$

By the inequality (3.17), we can write the inequality (3.20) as follows

$$
|y(t)-x(t)| \leqslant\left[|\Psi|(n \xi)\left(t^{\rho} / \rho\right)^{\gamma-1}+1\right] \delta \zeta_{h} h(t)+\left(\frac{\mu}{1-v}\right) \frac{\rho^{1-\alpha}}{\Gamma(\alpha)} \int_{0}^{t} \frac{\tau^{\rho-1}}{\left(t^{\rho}-\tau^{\rho}\right)^{1-\alpha}}|y(\tau)-x(\tau)| d \tau
$$

Finally, applying Lemma 3.11, we get

$$
|y(t)-x(t)| \leqslant\left(\left[|\Psi|(n \xi)\left(t^{\rho} / \rho\right)^{\gamma-1}+1\right]\left[1+\frac{C_{2} \mu \zeta_{h}}{(1-v)}\right] \zeta_{h}\right) \delta h(t):=\lambda_{\varphi} \delta h(t),
$$

where $C_{2}=C_{2}(\alpha)=\frac{1}{\Gamma(1+\alpha)}$ be a constant.

\section{Examples}

Example 4.1. Consider the following Hilfer-Katugampola fractional implicit differential equation

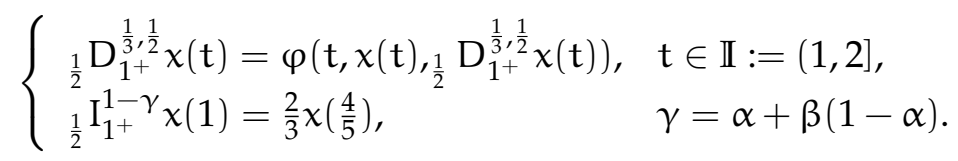

Here, $\rho=\frac{1}{2}>0, \alpha=\frac{1}{3}, \beta=\frac{1}{2}, \gamma=\frac{2}{3}, \xi=\frac{2}{3}, \eta=\frac{4}{5}$, and let

$$
\varphi\left(t, x(t)_{\frac{1}{2}} D_{1^{+}}^{\frac{1}{3}, \frac{1}{2}} \chi(t)\right)=\left(17 e^{2 t-1}\left[1+|x(t)|+\left.\right|_{\frac{1}{2}} D_{1^{+}}^{\frac{1}{3}, \frac{1}{2}} \chi(t) \mid\right]\right)^{-1}, \quad t \in \mathbb{I} .
$$


Clearly,

$$
\varphi(t, p, q)=\left(17 e^{2 t-1}[1+|p|+|q|]\right)^{-1}, \quad t \in \mathbb{I}, \quad p, q \in \mathbb{R}^{+}
$$

is a continuous function and for $p, q, \tilde{p}, \tilde{q} \in \mathbb{R}^{+}, t \in \mathbb{I}$, we have

$$
|\varphi(t, p, q)-\varphi(t, \tilde{p}, \tilde{q})| \leqslant \frac{1}{17 e}[|p-\tilde{p}|+|q-\tilde{q}|] .
$$

Thus, the suppositions $\left(\mathcal{H}_{2}\right)$ and $\left(\mathcal{H}_{3}\right)$ hold with $\mu=\nu=\frac{1}{17 e}$ and we can see that $|\Psi| \simeq 1.242338$ and $\Theta \simeq 0.067165<1$. From Theorem 3.2, it follows that the problem (4.1) has unique solution for $t \in \mathbb{I}$. Here, if we let $h(t)=t$, then we have

$$
{ }_{\frac{1}{2}} \mathrm{I}_{1^{+}}^{\frac{1}{3}} \mathrm{~h}(\mathrm{t})=\frac{(1 / 2)^{2 / 3}}{\Gamma(1 / 3)} \int_{1}^{t} \frac{\tau^{-1 / 2}(\tau)}{\left(\mathrm{t}^{1 / 2}-\tau^{1 / 2}\right)^{2 / 3}} \mathrm{~d} \tau \leqslant \frac{(1 / 2)^{2 / 3} \mathrm{t}}{\Gamma(1 / 3)} \int_{1}^{t} \frac{\tau^{-1 / 2}}{\left(t^{1 / 2}-\tau^{1 / 2}\right)^{2 / 3}} \mathrm{~d} \tau \leqslant \frac{6}{\Gamma(1 / 3)} \mathrm{h}(\mathrm{t}),
$$

so supposition $\left(\mathcal{H}_{4}\right)$ holds with $\zeta_{h} \simeq 2.239692$. Hence, from Theorem 3.13, it follows that the problem (4.1) is U.H.R.S..

Example 4.2. Consider the following Hilfer-Katugampola fractional implicit differential equation

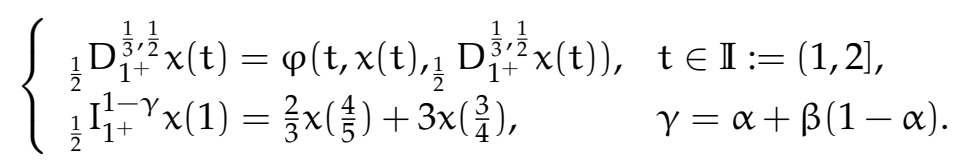

In addition of previous example, we have $\xi_{1}=\frac{2}{3}, \eta_{1}=\frac{4}{5}, \xi_{2}=3, \eta_{2}=\frac{3}{4}$, and let

$$
\varphi\left(t, x(t)_{, \frac{1}{2}} D_{1^{+}}^{\frac{1}{3}, \frac{1}{2}} \chi(t)\right)=\frac{1}{107 e^{3 t+2}}\left(\frac{|x(t)|}{1+|x(t)|}+\frac{\left|{ }_{\frac{1}{2}} D_{1^{+}}^{\frac{1}{3}, \frac{1}{2}} \chi(t)\right|}{1+||_{\frac{1}{2}} D_{1^{+}}^{\frac{1}{3}, \frac{1}{2}} \chi(t) \mid}\right), \quad t \in \mathbb{I} .
$$

Clearly, for any $p, q \in \mathbb{R}^{+}$, we have

$$
\varphi(t, p, q)=\frac{1}{107 e^{3 t+2}}\left(\frac{p}{1+p}+\frac{q}{1+q}\right)
$$

is a continuous function satisfies the hypotheses of Theorem 3.1 and for $p, q, \tilde{p}, \tilde{q} \in \mathbb{R}^{+}$and $t \in \mathbb{I}$, we have

$$
|\varphi(t, p, q)-\varphi(t, \tilde{p}, \tilde{q})| \leqslant \frac{1}{107 e^{5}}[|p-\tilde{p}|+|q-\tilde{q}|] .
$$

Thus, the suppositions $\left(\mathcal{H}_{2}\right)$ and $\left(\mathcal{H}_{3}\right)$ hold with $\mu=\nu=\frac{1}{107 e^{5}}$ and we can see that $|\Psi| \simeq 0.5906272$ and $\Theta \simeq 0.0003054<1$. Hence, from Theorem 3.2, it follows that the problem (4.2) has unique solution for $\mathrm{t} \in \mathrm{I}$. Furthermore, it implies from Theorem 3.12, that the problem (4.2) is U.H.S..

\section{Acknowledgment}

The authors are grateful to the referee for his/her important remarks and suggestions.

\section{References}

[1] S. Abbas, M. Benchohra, J. E. Lagreg, A. Alsaedi, Y. Zhou, Existence and Ulam stability for fractional differential equations of Hilfer-Hadamard type, Adv. Difference Equ., 2017 (2017), 14 pages. 1

[2] S. Abbas, M. Benchohra, G. M. N'Guerekata, Topics in fractional differential equations, Developments in Mathematics, Springer, New York, (2012). 1 
[3] R. Almeida, Variational problems involving a Caputo-type fractional derivative, J. Optim. Theory Appl., 174 (2017), 276-294. 1

[4] Sz. Andras, J. J. Kolumban, On the Ulam-Hyers stability of first order differential systems with nonlocal initial conditions, Nonlinear Anal. Theory Methods Appl., 82, (2013), 1-11. 1

[5] D. Baleanu, Z. B. Guvenc, J. A. Tenreiro Machado (Eds.), New trends in nanotechnology and fractional calculus applications, Springer, New York, (2010). 1

[6] M. Benchohra, J. E. Lazreg, Nonlinear fractional implicit differential equations, Commun. Appl. Anal., 17 (2013), 471-482. 1

[7] M. Benchohra, J. E. Lazreg, On stability for nonlinear fractional implicit differential equations, Matematiche (Catania), 70 (2015), 49-61.

[8] M. Benchohra, J. E. Lazreg, Existence and Ulam stability for nonlinear fractional implicit differential equations with Hadamard derivative, Stud. Univ. Babes-Bolyai Math., 62 (2017), 27-38.

[9] S. P. Bhairat, Existence and stability of fractional differential equations involving generalized Katugampola derivative, Stud. Univ. Babes-Bolyai. Math., 65 (2020), 29-46. 1

[10] S. P. Bhairat, On stability of generalized Cauchy-type problem, Dyn. Contin. Discrete Impuls. Syst. Ser. A Math. Anal., 27 (2020), 235-244. 1

[11] R. Caponetto, G. Dongola, L. Fortuna, I. Petras, Fractional order systems: modeling and control applications, World scientific publishing com. Pte. Ltd., (2010). 1

[12] A. Carpinteri, F. Mainardi, Fractals and fractional calculus in continuum mechanics, Springer-Verlag, Vienna, (1997).

[13] S. Das, Functional fractional calculus, Springer, Berlin, (2011). 1

[14] A. Granas, J. Dugundji, Fixed Point Theory, Springer, New York, NY., (2003). 2.4, 2.7

[15] R. Hilfer, Applications of Fractional Calculus in Physics, World Scientific Publishing Co., Inc., River Edge, NJ, (2000). 1

[16] D. H. Hyers, On the stability of the linear functional equation, Proc. Nat. Acad. Sci. U.S.A., 27 (1941), 222-224. 1

[17] R. W. Ibrahim, Generalized UlamHyers stability for fractional differential equations, Int. J. Math., 23 (2012). 1

[18] R. W. Ibrahim, S. Harikrishnan, K. Kanagarajan, Existence and stability of Langevin equations with two HilferKatugampola fractional derivatives, Stud. Univ. Babes-Bolyai Math., 63 (2018), 291-302. 1, 1

[19] S.-M. Jung, Hyers-Ulam stability of linear differential equations of first order, Appl. Math. Lett., 17 (2004), 1135-1140. 1

[20] S.-M. Jung, Hyers-Ulam stability of linear differential equations of first order II, Appl. Math. Lett., 19 (2006), $854-858$.

[21] S.-M. Jung, Hyers-Ulam-Rassias stability of functional equations in nonlinear analysis, Springer, New York, (2011). 1

[22] U. N. Katugampola, New approach to a generalized fractional integral, Appl. Math. Comput., 218 (2011), 860-865. 1, 2.7

[23] U. N. Katugampola, A new approach to generalized fractional derivatives, Bull. Math. Anal. Appl., 6 (2014), 1-15. 1

[24] A. A. Kilbas, H. M. Srivastava, J. J. Trujillo, Theory and applications of fractional differential equations, Elsevier Science B.V., Amsterdam, (2006). 1

[25] F. Mainardi, Fractional calculus and waves in linear viscoelasticity, An introduction to mathematical models, Imperial College Press, London, (2010). 1

[26] P. Muniyappan, S. Rajan, Hyers-Ulam-Rassias stability of fractional differential equation, Int. J. Pure Appl. Math., 102 (2015) 631-642. 1

[27] D. S. Oliveira, E. C. de Oliveira, Hilfer-Katugampola fractional derivative, Comput. Appl. Math., 37 (2018), $3672-3690$. $1,2,2.2,2.3,2.5,2.6,2$

[28] I. Podlubny, Fractional differential equations, Academic Press, San Diego, (1999). 1

[29] Th. M. Rassias, On the stability of the linear mapping in Banach spaces, Proc. Amer. Math. Soc., 72 (1978), 297-300. 1

[30] Th. M. Rassias, J. Brzdek, Functional equations in mathematical analysis, Springer, New York, (2012). 1

[31] I. A. Rus, Ulam stabilities of ordinary differential equations in a Banach space, Carpathian J. Math., 26 (2010), $103-107$. 1

[32] J. Sabatier, O. P. Agrawal, J. A. Tenreiro Machado (Eds.), Advances in fractional calculus, Springer, Dordrecht, (2007). 1

[33] A. Y. A. Salamooni, D. D. Pawar, Unique positive solution for nonlinear Caputo-type fractional q-difference equations with nonlocal and Stieltjes integral boundary conditions, Fract. Differ. Calc., 9 (2019), 295-307. 1

[34] A. Y. A. Salamooni, D. D. Pawar, Existence and continuation of solutions of HilferKatugampola-type fractional differential equations, arXiv, 2020 (2020), 19 pages. 3.11

[35] S. M. Ulam, A collection of mathematical problems, Interscience Tracts in Pure and Applied Mathematics, Interscience Publishers, New York, (1960). 1

[36] S. M. Ulam, Problems in modern mathematics, Science Editions John Wiley \& Sons, Inc., New York, (1964). 1

[37] D. Vivek, K. Kanagarajan, E. M. Elsayed, Some existence and stability results for hilferfractional implicit differential equations with nonlocal conditions, Mediterr. J. Math., 15 (2018). 1

[38] J. Wang, L. Lv, Y. Zhou, Ulam stability and data dependence for fractional differential equations with Caputo derivative, Electron. J. Qual. Theory Differ. Equ., 2011 (2011), 10 pages. 1

[39] J. Wang, L. Lv, Y. Zhou, New concepts and results in stability of fractional differential equations, Commun. Nonlinear Sci. Numer. Simul., 17 (2012), 2530-2538. 1 